\title{
Singularity, complexity, and quasi-integrability of rational mappings
}

\author{
G. Falqui* and C.-M. Viallet \\ July 1992 \\ Laboratoire de Physique Théorique et des Hautes Energies \\ Unité Associée au CNRS (URA 280) \\ Université Paris VI Boîte 126 \\ Tour $16-1^{\text {er }}$ Etage / 4 Place Jussieu \\ 75252 PARIS CEDEX 05
}

\begin{abstract}
We investigate global properties of the mappings entering the description of symmetries of integrable spin and vertex models, by exploiting their nature of birational transformations of projective spaces. We give an algorithmic analysis of the structure of invariants of such mappings. We discuss some characteristic conditions for their (quasi)-integrability, and in particular its links with their singularities (in the 2-plane). Finally, we describe some of their properties qua dynamical systems, making contact with Arnol'd's notion of complexity, and exemplify remarkable behaviours.
\end{abstract}

PAR-LPTHE 92/26 To appear in Communications in Mathematical Physics

*Supported in part by Ministère de la Recherche et de la Technologie 


\section{Introduction}

We want to analyze in detail some realizations of Coxeter groups [1, 2] by birational transformations of projective spaces which have been shown to appear in the description of the symmetries of quantum integrable systems [3, 4 , 同] [6, 7, 8, 9, 10.

The first motivation to look at these realizations resides of course in their relations with the star-triangle and the Yang-Baxter equations or their higher dimensional generalizations such as the tetrahedron equations. A characteristic feature of the orbits of the known solutions of the Yang-Baxter equations under these groups is that they are confined to subvarieties of high codimension of the parameter space (actually curves), signaling the existence of an unexpectedly large number of algebraically independent invariants. The discovery and the analysis of the possible invariants is a decisive step in the study of the YangBaxter (tetrahedron,...) equations, in particular for what concerns the so-called baxterization problem [11.

Another motivation is to use these realizations to construct discrete time evolution maps, as it is usual in the study a la Poincaré of dynamical systems, by iterating some element of the group. One of the main questions in this setting is again to bring to the light the possible presence of invariants and invariant tori 12, 13, $14 \sqrt[15]{15}, 16,17,18,19] 6,0,8,9,10$.

If a realization admits algebraic invariants, we will say it has a property of quasi-integrability.

In 6, 7] it was shown, among other things how the direct graphical investigation of the group action by means of numerical calculations ("drawing the picture") leads to a nice representation of the orbits. We want to elaborate on the problem and discuss the deeper structure of the non linear realizations by birational transformations of projective spaces.

We first recall which kind of infinite Coxeter groups arise in the theory of solvable models of statistical mechanics and collect some facts about birational maps which we shall use in the sequel.

We then analyze the cohomological structure of the possible invariants. We give the general (albeit formal) solution to the problem in terms of an algorithmic research of linear systems on $\mathbb{P}_{n}$ satisfying a covariance property with respect to the realizations we handle. We then specialize to the case $n=2$, analyze the singular locus of the group, and discuss some illustrative examples.

In the final section we make contact with Arnol'd's notion of complexity [20, 21], which measures the growth of the topological non-triviality of the intersection of a fixed subvariety and the image of another one under an iteration map. We will argue how our notion of quasi-integrability is related to a polynomial growth of the complexity while the generic one is exponential. 


\section{Coxeter groups and birational realizations}

One of the outcomes of [6]-[10] is the construction of a number of groups generated by involutions and of various rational realizations on projective spaces.

Consider the Coxeter group $G$ engendered by $\nu$ involutions $I_{1}, I_{2}, \ldots, I_{k}, \quad(k=$ $1 \ldots \nu)$, verifying no relations other than the involution property. The group $G$ is infinite and there are two essentially different situations.

If $\nu=2$, the group is the infinite dihedral group $\mathbb{Z}_{2} \ltimes \mathbb{Z}$, and all elements may uniquely be written $I_{1}^{\alpha}\left(I_{1} I_{2}\right)^{q}$, with $\alpha=0,1$ and $q \in \mathbb{Z}$. The number of elements of given lenght $l$ is 2 .

If $\nu \geq 3$, the number of elements of lenght $l$ grows exponentially with $l$, and the group is in a sense bigger (still countable).

As an example for the groups described in $[6]-[10]$, the number $\nu$ of generators depends on the dimension $d$ of the lattice: it is just $2^{d-1}$ so that if $d=2, G$ is generated by two involutions and if $d \geq 3, G$ is generated by more than three involutions.

One may then construct various realizations $\Gamma$ of $G$ by explicit transformations of some projective space. They are obtained by specifying the realization of the generators. Since it is precisely the realizations that we want to study here, and especially the problem of the existence of invariants, we will mainly talk about $\Gamma$ and not $G$, and use the same notation $I_{k}$ for the generators of $G$ and their representatives in $\Gamma$.

The realizations $\Gamma$ we consider are essentially obtained from operations on matrices, especially matrix inversions, and transpositions of their entries, the matrices being originally matrices of Boltzmann weigths of statistical mechanical spin and vertex models on the lattice or $R$-matrices of 2-dimensional field theories. The projective space we consider is just the space of entries of the matrices up to a common multiplicative factor.

Let us describe here typical examples of such realizations.

Suppose $m$ is a $q \times q$ matrix. The ordinary matrix inverse $I$ defines an involutive rational transformation of $\mathbb{P}_{q^{2}-1}$, which reads in homogeneous coordinates:

$$
I: \quad m_{i j} \longrightarrow \text { cofactor of } m_{i j}
$$

We may also consider the element by element inverse (so called Hadamard inverse):

$$
J: \quad m_{i j} \longrightarrow 1 / m_{i j}
$$

These two inverses appear in the study of spin models. Notice that $I^{2}=J^{2}=1$ and there is no other relation between $I$ and $J$. In particular $I$ and $J$ do not commute and $\varphi=I J$ is of infinite order.

It is of course possible to define all kinds of block inverses, the size of the blocks ranging from the full matrix size (for $I$ ) and 1 (for $J$ ).

One may also mix $I$ with linear transformations. This happens already when $I$ and $J$ defined by (11) and (2) are collineated, i.e when there exists a linear 
transformation $C$ such that $I=C^{-1} J C$ (see [6]). We may also mix $I$ with permutations of the entries.

Let us describe here the transpositions of entries which appear in the study of vertex models on a $d$-dimensional lattice 9,10$]$.

Suppose $M$ is a multiindex matrix of size $q^{d} \times q^{d}$ written in the form $M_{j_{1} j_{2} \ldots j_{d}}^{i_{1} i_{2} \ldots i_{d}}$. There exist $d$ different partial transpositions $t_{1}, t_{2}, \ldots, t_{d}$ with the evident definition:

$$
\left(t_{k} M\right)_{j_{1} \ldots j_{k} \ldots j_{d}}^{i_{1} \ldots i_{k} \ldots i_{d}}=M_{j_{1} \ldots i_{k} \ldots j_{d}}^{i_{1} \ldots j_{k} \ldots i_{d}} .
$$

We clearly have a product and an inverse $I$ for these multiindex matrices. We may define $2^{(d-1)}$ new inversions by:

$$
I_{\kappa}=t_{\alpha_{1}} \ldots t_{\alpha_{s}} I t_{\alpha_{s+1}} \ldots t_{\alpha_{d}}=t_{\alpha_{s+1}} \ldots t_{\alpha_{d}} I t_{\alpha_{1}} \ldots t_{\alpha_{s}}
$$

where $\kappa=\left(\left\{\alpha_{1}, \ldots \alpha_{s}\right\},\left\{\alpha_{s+1}, \ldots \alpha_{d}\right\}\right)$ is a partition of $\{1, \ldots, d\}$.

These various inverses yield involutive (bi)rational mappings of $\mathbb{P}_{q^{2 d}-1}$. They are related by collineations, easy to write from the representation of the partial transpositions. Note that the product of all $t_{k}$ 's is the full transposition $t$ and commutes with all the inverses. Such realizations appear in the study of vertex models.

We may further enrich the representations by imposing constraints on the entries of the matrices, provided the transformations are compatible with these constraints (see [6] for the notion of admissible patterns). This yields realizations on projective spaces of lower dimensions.

Needless to say that along these ideas, one may construct a variety of involutions acting on various projective spaces.

We stress that, at the level of the realization, there may exist additional relations between the generators, possibly making it finite (as in example (6.4)).

\section{Some facts about rational mappings}

In this section we collect some results about rational and birational mappings between algebraic varieties (see for example [22, 23, 24, 25]).

Definition 3.1 A correspondence $\varphi_{Z}: X \rightarrow Y$ between algebraic varieties $X$ and $Y$ is an algebraic subset $Z \subset X \times Y . \varphi_{Z}$ is a rational map if there is a Zariski open set $U \subset X$ on which the correspondence is one to one.

If $Z \subset X \times Y$ is a rational map, the inverse correspondence is defined by the graph $Z^{-1}:=\{(y, x) \in Y \times X \mid(x, y) \in Z\}$. If the correspondences $Z$ and $Z^{-1}$ are both rational mappings, then $Z$ (or $\varphi_{Z}$ ) is called a birational transformation. A birational map is a biholomorphism except on subvarieties of codimension at least two. 
A linear system $D$ on $X$ is a non empty linear subspace of the space of global sections of some line bundle $\mathcal{L}$ over $X$. Its base locus is the set of common zeroes of all sections in $D$. A remarkable result is that 23] there exists a one to one correspondence between linear systems on $X$ of dimension $d$ with base locus of codimension not less that 2 and rational maps $X \stackrel{\phi}{\longrightarrow} \mathbb{P}_{d-1}$ up to projective automorphisms of $\mathbb{P}_{d-1}$.

For what we are concerned with, the paradigm of rational map is the socalled $\sigma$-process or blow up of a point.

We refer to [24, 25] for the general definitions. We shall call Hadamard inversion and generically denote by $J$ the prototypical birational mapping in $\mathbb{P}_{n}$. Let $x_{0}, x_{1}, \ldots, x_{n}$ and $y_{0}, y_{1}, \ldots, y_{n}$ be coordinates in two different copies of $\mathbb{P}_{n}$ and let us consider the algebraic set $Z \subset \mathbb{P}_{n} \times \mathbb{P}_{n}$ given by the $n$ equations $x_{0} y_{0}=x_{1} y_{1}=\ldots=x_{n} y_{n}$. By definition, the graph of $J$ is $Z$.

It is valuable to specialize to $n=2$ : outside the triangle $x_{0} x_{1} x_{2}=0, Z$ is the graph of the map $\left[x_{0}, x_{1}, x_{2}\right] \sim\left[1 / x_{0}, 1 / x_{1}, 1 / x_{2}\right]$. The generic point on the line $x_{i}=0$ is sent into the point $p_{i}$ whose only non-vanishing coordinate is $y_{i}$, while to the points $p_{i}$ corresponds the entire line $y_{i}=0$. One can say that $J$ blows up $p_{i}$ to the line $x_{i}=0$ and blows down the line $x_{i}=0$ to the point $p_{i}$.

Finally, we recall the following properties, which will be used in what follows. From the description of a birational mapping as an algebraic set in the product $X \times Y$ it is apparent that $\varphi$ blows up $p$ to a divisor $D$ if and only if $\varphi^{-1}$ blows down $D$ to $p$, and it is also evident that different points $p_{1}$ and $p_{2}$ cannot be blown up to the same divisor $D$ (otherwise the inverse map would not be rational).

Also, it is a standard result that blowing up a point adds a free factor $\mathbb{Z}$ in the Picard group of $X$. It follows that if $\varphi$ is a birational map in $\mathbb{P}_{2}$ which blows up $n$ points, then it must blow down exactly $n$ exceptional divisors, as explained above.

\section{Invariants and quasi-integrability}

Let $\Gamma$ be a group of birational transformations in $\mathbb{P}_{n}$. A meromorphic function $\Delta: \mathbb{P}_{n} \rightarrow \mathbb{P}_{1}$ deserves to be called a $\Gamma$-invariant if it satisfies

$$
\Delta(g(x))=\Delta(x) \quad \forall g \in \Gamma
$$

Since a meromorphic function on an algebraic variety can be thought of as the ratio of two sections of a suitable line bundle, we are naturally lead to the following scheme.

A $\Gamma$ one-cocycle is a collection of sections $a(g, x)$ of some line bundles over $\mathbb{P}_{n}$ satisfying the cocycle condition:

$$
a\left(g_{1} g_{2}, x\right)=a\left(g_{1}, g_{2} x\right) \cdot a\left(g_{2}, x\right)
$$


A section $\sigma$ of a line bundle will be called $a$-covariant (for some cocycle a) if the equation

$$
\sigma(g x)=a(g, x) \cdot \sigma(x)
$$

holds. This equation may be reformulated in group cohomology terms [26] as $a=\delta \sigma$ meaning that $a$ is actually a coboundary.

Finding a $\Gamma$-invariant is equivalent to finding two sections $\sigma_{1}$ and $\sigma_{2}$ verifying equation (6) for the same $a$. This means that we are interested in the coboundaries of the 0 -cochains rather than the cohomology groups. The strategy is to find 1-cocycles admitting a sufficient number of primitives.

As a side remark, we notice that these equations, which will play a prominent role in the sequel, are well defined at all points of $\mathbb{P}_{n}$. In fact for any birational transformation $g$, the singular locus is a subvariety of codimension greater than or equal to two in $\mathbb{P}_{n}$, and the equations above admit a unique holomorphic extension to the whole of $\mathbb{P}_{n}$, even if they have meaning in the point set sense only on the nonsingular locus of $g$.

We are interested in the case where $\Gamma$ is a Coxeter group, i.e. is generated by $\nu$ involutions $I_{k}$ of degree $d_{k}$ (the degree is a natural notion in terms of the homogeneous coordinates $\left.\left[x_{0}, \ldots, x_{n}\right]\right)$. A $\Gamma$ one-cocycle will be completely specified by the assignment of $\nu$ sections $a\left(I_{k}, x\right)$. Remarkably, the possible values of $a\left(I_{k}, x\right)$ may be found explicitely.

Each involution $I_{k}$ defines a characteristic polynomial $\phi_{k}$ of degree $d_{k}^{2}-1$ in the following manner. The $I_{k}$ being involutions, $I_{k}^{2}$ appears as the multiplication by a degree $d_{k}^{2}-1$ polynomial $\phi_{k}\left(x_{0}, \ldots, x_{n}\right)$. We then have the following

Lemma 4.1 If $a(g, x)$ is a trivial $\Gamma$ cocycle, the sections $a\left(I_{k}, x\right)$ divide a suitable power of $\phi_{i}$ for $i=1, \ldots, \nu$.

Proof. Suppose $a=\delta \sigma$. Then, from the coboundary equation (6) for $g=I_{i}^{2}$ we get that $a\left(I_{i}^{2}, x\right)=\phi(x)^{m}$, with $m=\operatorname{deg}(\sigma)$. The assertion follows from the cocycle condition (5).

Definition 4.1 We shall say that $\Gamma$ is collineated when its generators are all conjugated by means of elements of $P G L(n+1, \mathbb{C})$ to a standard one, $K$.

This is the case for a number of models among which the Baxter model $(n=3)$ and the examples of section 6 .

If the characteristic polynomial $\phi_{K}$ factorizes into $\phi_{K}=\prod_{l=0}^{j} p_{l}^{d_{l}}$, then it is clear that for every $i$ there exist a set of global homogeneous coordinates $\left[X_{0}^{(i)}, \ldots, X_{n}^{(i)}\right]$ in which $a\left(I_{i}, x\right)$ will be the product of the same polynomials $p_{l}$, possibly weighted with different exponents $d_{l}^{\prime}$.

Remark. When $K$ is the Hadamard inversion $J$ in $\mathbb{P}_{n}$ a straightforward computation shows that $\phi_{J}=\prod_{l=0}^{n} x_{l}^{(n-1)}$ so that we get

Proposition 4.1 A coboundary for a collineated Coxeter group of birational transformations with generators conjugated to the Hadamard inversion $J$ in $\mathbb{P}_{n}$ is a monomial in some suitable homogeneous coordinates, with coefficient \pm 1 . 
It is apparent that our cohomological setting gives an algorithmic prescription for the search for invariants: find first the characteristic polynomials $\phi_{k}$, which is straightforward, then the possible coboundaries, which is a factorization problem, and check how many primitives they have, which amounts to solving a linear problem.

The realization $\Gamma$ in $\mathbb{P}_{n}$ admits $p$ invariants $\Delta_{1}, \ldots \Delta_{p}$ if there exists an $a$-covariant linear system $L_{p}^{d}$ of projective dimension $p$ and degree $d$ for some cocycle $a$. It is not guaranteed that the orbits of a realization admitting $p$ invariants lie on subvarieties of dimension $n-p$. Indeed the question of the algebraic independence of the invariants has to be examined further [27].

One has to realize how exceptional is the existence of any invariant of the birational realization, as the following argument shows.

Let us consider covariance with respect to one generator $K$ of $\Gamma$. Suppose $K$ is of degree $d_{K}$, and suppose we are looking for an invariant of degree $m$. Clearly from equation (6) the degree $q$ of the cocycle is related to $d_{K}$ and $m$ by $m\left(d_{K}-1\right)=q$. The dimension of the space $\mathcal{P}(m, n)$ of homogeneous polynomials of degree $m$ in $n$ variables is $\left(\begin{array}{c}n+m-1 \\ m-1\end{array}\right)$. The requirement of $K_{-}$ covariance selects a linear system $L^{K}$ in $\mathcal{P}(m, n)$, of generic dimension at most $\frac{1}{2}\left(\begin{array}{c}n+m-1 \\ m-1\end{array}\right)$. Imposing the same condition for another generator $I$ leads to look for the intersection of hyperplanes of at most complementary dimensions in $\mathcal{P}(m, n)$. This intersection is generically empty. As a consequence we have

Proposition 4.2 The action of a generic Coxeter group of birational transformations in $\mathbb{P}_{n}$ does not admit any non-trivial invariant.

In the particular case of collineated realizations, this leads to the further property, which we prove for $\nu=2$ for simplicity.

Proposition 4.3 The set of collineated groups admitting a non-trivial invariant has the structure of a quasi-projective variety.

Proof. The group $P G L(n+1, \mathbb{C})$ admits a natural structure of quasi-projective variety, since it is identified with the complement of the degree $(n+1)$ hypersurface $\operatorname{det} A=0$ in $\mathbb{P}^{(n+1)^{2}-1}$. Choosing $K$ as prototypical generator of $\Gamma, I$ is specified (in the collineated case) by the choice of an element $C \in P G L(n+1, \mathbb{C})$ by $I=C^{-1} K C$. If for any polynomial $P$ we define $P_{C}:=P\left(C^{-1} x\right)$ and set $y=C x$, then the covariance equation with respect to $I$ reads

$$
P_{C}(K y)=a_{I}\left(C^{-1} y\right) P_{C}(y)
$$

For each possible cocycle $a$, this is an algebraic equation in the parameters $t_{a}$ of the matrix $C$. The coordinates of any basis $\left\{e_{\alpha}\right\}$ in $L^{I}$ with respect to a basis in $\mathcal{P}(m, n)$ completed from a basis of $L^{K}$ may be expressed as algebraic functions 
of the $t_{a}$ 's. The condition of nontriviality of the intersection is a condition on the rank of the matrix

$$
B:=\left[\begin{array}{cc}
\mathbf{1} & \mathbf{0} \\
e_{\alpha}^{i}\left(t_{1}, . . t_{(n+1)^{2}-1}\right) & \ldots
\end{array}\right]
$$

which in turn is an algebraic condition on the $e_{\alpha}^{i}\left(t_{1}, . . t_{(n+1)^{2}-1}\right)$, concluding the proof.

\section{Realizations in the 2-plane}

Let us discuss mappings in $\mathbb{P}_{2}$. Here our analysis is made quite complete by the fact that all rational maps from an algebraic surface can be described in terms of $\sigma$ processes and also that singularities of birational maps can occur only at points. Moreover a theorem of M. Noether [25] assures that the group of birational maps is generated by the inversion $J$ together with the projective group $P G L(3, C)$. We will restrict ourselves here to $\nu=2$, i.e. $\Gamma$ is generated by two involutions $I$ and $K$.

Definition 5.1 The singular locus of $\Gamma, S(\Gamma)$ is defined as

$$
S(\Gamma)=\left\{x \in \mathbb{P}_{2} \mid \exists g \in \Gamma \text { s.t. } g \text { blows } x \text { up }\right\}
$$

Since $\Gamma$ is generated by $I$ and $K, S(\Gamma)$ is obtained by the action of $\Gamma$ on the singular points of $I$ and $K$.

Definition 5.2 We will say that $\Gamma$ is properly singular if $S(\Gamma)$ contains at least four points in general position, i.e. such that no three of them are aligned.

Let $\Pi(\Gamma)$ be the set of singular divisors:

$$
\Pi(\Gamma)=\left\{\pi \in \operatorname{Div}\left(\mathbb{P}_{2}\right) \mid \exists g \in \Gamma \text { s.t. } g \text { blows } \pi \text { down to a point }\right\}
$$

Let us suppose that $\Gamma$ admits a rational invariant $\Delta$ and let us look at what happens at points in $S(\Gamma)$. The equation $\Delta(g(x))=\Delta(x)$ is clearly meaningless at the indetermination points of $\Delta$. Noticing that $\forall x \in S(\Gamma), \exists \pi_{x} \in \Pi(\Gamma)$ such that $\Pi_{x}$ is blown down to $x$ by some element $g \in \Gamma$, we can conclude that $x \in S(\Gamma)$ is either an indetermination point of $\Delta$ or $\Delta$ is constant along the corresponding divisor $\pi_{x}$, i.e. $\pi_{x}$ belongs to the pencil of curves $\Delta=$ const. Then we can state the

Proposition 5.1 If $S(\Gamma)$ is infinite, and $\Gamma$ is properly singular, then $\Gamma$ does not admit any invariant. 
Proof. With the above observation in mind, the only case we must rule out is that $\Gamma$ admits an infinite number of singular points and only a finite number of singular divisors, since by Bezout's theorem, any rational degree $d$ invariant admits at most $d^{2}$ indetermination points. Let us suppose that $\# S(\Gamma)=\infty$ and $\# \Pi(\Gamma)=N$ and let us consider the set $\mathcal{P}=\left\{\Pi_{\alpha}\right\}\left(\alpha=1 \ldots 2^{N}\right)$ of parts of $\Pi(\Gamma)$. We can make a partition of $\Gamma$ into $2^{N}$ disjoint subset $\Gamma=\bigcup_{\alpha=1}^{N} \Gamma_{\alpha}$, where

$$
\Gamma_{\alpha}=\left\{g \in \Gamma \mid g \text { blows down exactly all the divisors in } \Pi_{\alpha}\right\}
$$

At least one of the $\Gamma_{\alpha}$, say $\Gamma_{0}$ is infinite. If we then consider any pair of elements $\left(h_{0}, l_{0}\right)$ in $\Gamma_{0}$, the product $h_{0} \cdot l_{0}^{-1}$ is a birational map without singularities, and hence an element $D \in P G L(3, \mathbb{C})$. Since $\Gamma_{0}$ is infinite, we can arrange things so that $D$ is a non trivial word of even lenght. Since words of odd length in $\Gamma$ are involutions one has:

$$
D K D=K \quad D I D=I
$$

It follows that $D$ permutes the points of $S(\Gamma)$, and some power $D^{k}$ must be the identity. As a consequence, there is some non-trivial product $(I K)^{l}=\mathbf{1}$, meaning that actually $\Gamma$ is finite, and contradicting the infiniteness of $S(\Gamma)$.

Remark. The above proposition proves that a necessary condition for existence of an invariant is the finiteness of the singular locus. It is tempting to conjecture that this is also a sufficient condition for any properly singular realization. This is unfortunately not the case, as the example of section (6.6) will show.

The next step is to try to relate the singular locus of the group with the singular locus of the would-be invariant. Let the $\Delta$ be a rational invariant for $\Gamma$ and let $S(\Delta)$ be the set of its indetermination points. If $y \in S(\Delta)$ and $y \notin S(\Gamma)$, the (finite) orbit $\Gamma_{y}$ of $y$ is made out of indetermination points of $\Delta$, on which every element $g \in \Gamma$ is regular. Since $\Gamma_{y}$ is of even order, say $2 l, y$ is a fixed point for $(I K)^{n \cdot l}, \quad n \in \mathbb{Z}$. Let $\tilde{\mathbb{P}}_{2}^{y} \stackrel{p r}{\longrightarrow} \mathbb{P}_{2}$ be the 2-plane $\mathbb{P}_{2}$ blown up at $y$. Since we are working with $\mathbb{P}_{2}, \Delta$ extends to a function $\tilde{\Delta}$ on $\tilde{\mathbb{P}}_{2}^{y}$ with no indetermination points in a neighbourhood of the exceptional divisor $E=p r^{-1}(y)$. The restriction $\varphi=\left.\tilde{\Delta}\right|_{E}$ is a meromorphic map on $\mathbb{P}_{1}$ whose value is determined by the limits of $\Delta(x)$ along lines in $\mathbb{P}_{2}$ passing through $y$.

We will say that $\Gamma$ is non-degenerate if for every $g \in \Gamma$ the tangent map to $g$ is non-nilpotent at every isolated fixed point of $g$. Then we can state the

Proposition 5.2 Let $\Gamma$ be a non-degenerate Coxeter group of birational transformations of $\mathbb{P}_{2}$. If $\Gamma$ admits an invariant $\Delta$, then $S(\Delta) \subset S(\Gamma)$.

Proof. Suppose $y \in S(\Delta)$ and $y \notin S(\Gamma)$; the non-degeneracy of $\Gamma$ ensures that there is at least one line in $P T_{\mathbb{P}_{2}}(y)$ whose orbit under the tangents to $(I K)^{n \cdot l}, \quad l \in \mathbb{Z}$ is infinite (recall that $(I K)^{l}$ is a minimal length element in $\Gamma$ which fixes $y$ ). Hence $\varphi$ as defined above assumes the same value on an infinite number of points, i.e. it is constant over the whole of $E_{y}$. But this in turn 
implies that $\Delta$ is well defined at $y$ which contradicts $y \in S(\Delta)$ and ends the proof.

Notice that the converse inclusion $S(\Gamma) \subset S(\Delta)$ does not hold in general. If the inclusion is strict, we have seen that for any $x \in S(\Gamma)$ and not in $S(\Delta)$, the invariant $\Delta$ is constant along some divisor $\pi_{x}$. This gives a useful information about the invariant (see example 6.3).

As for what the singularities of the generic curve $\Sigma_{\lambda}$ of the pencil are related to the singular orbit $S(\Gamma)$ the following considerations hold. By Bertini's theorem, generic curves are smooth outside the base locus $S(\Delta)$ whence the chain of inclusions

$$
S\left(\Sigma_{\lambda}\right) \subset S(\Delta) \subset S(\Gamma)
$$

Moreover, exploiting the genus formula for singular curves one can give relations 7 between the degree $d$ of the invariant and $S(\Gamma)$. If $\Sigma$ is a degree $d$ curve in $\mathbb{P}_{2}$ with singular locus $\operatorname{Sing}(\Sigma)$, then [22]

$$
g(\Sigma)=\frac{(d-1) \cdot(d-2)}{2}-\sum_{p \in \operatorname{Sing}(\Sigma)} \delta_{p}
$$

with $\delta_{p}$ depending on the type of the singularity at p. Since $\Sigma_{\lambda}$ is irreducible and admits $\Gamma$ as an infinite group of automorphisms, thanks to the inclusion (8) and to the fact that $\delta_{p}=0$ if $p$ is not in $\operatorname{Sing}\left(\Sigma_{\lambda}\right)$, one has

$$
\frac{(d-1) \cdot(d-2)}{2}-\sum_{p \in S(\Gamma)} \delta_{p}=\left\{\begin{array}{l}
0 \\
1
\end{array}\right.
$$

This relation shows that there is a balance between the degree of the invariants and the number and nature of the singular points of the generic curve, and consequently of $\Gamma$.

\section{$6 \quad$ Examples}

We describe here some specific examples in $\mathbb{P}_{2}$ with two generators $I$ and $J$. The physical origin of the models we will be dealing with is to be found notably among two-dimensional spin models with interaction along the edges [3], and this explains the terminology we use.

We fix $J$ to be the Hadamard inversion $\left.\left[x_{i}\right] \sim \sim 1 / x_{i}\right]$, and $I$ to be $I=$ $C^{-1} J C$ with the collineation matrix $C \in P G L(3, \mathbb{C})$. We will concentrate on the parametrization by $C$ and examine some algebraic families in $P G L(3, \mathbb{C})$. For some of them $\Gamma$ admits a non trivial algebraic invariant.

We can associate to $\Gamma$ a diagram $D_{\Gamma}$ whose vertices are the points in $S(\Gamma)$ and where two vertices $p_{1}$ and $p_{2} \in S_{\Gamma}$ are joined by an edge if either $p_{1}=J p_{2}$

\footnotetext{
${ }^{1}$ We thank M. Talon for useful remarks on this point.
} 
or $p_{1}=I p_{2}$. The edges are oriented if $I$ (resp. $J$ ) can be applied in one direction only. The diagram does not characterize the examples, but is a useful tool. In particular the families we give here have been obtained by deformations of given collineation, demanding the stability of the topology of the diagram.

The singular points of $J$ are $P_{1}=[1,0,0], P_{2}=[0,1,0], P_{3}=[0,0,1]$, and the one of $I$ are $Q_{i}=C^{-1}\left(P_{i}\right),(i=1,2,3)$.

One should notice that a number of the families we produce in this way fall into the general form found in [28] (i.e. verifying $C([1,0,0])=[1,1,1]$ and $C^{2}([1,0,0]=[1,0,0])$, but with non integer entries. This general form depends on four parameters:

$$
\left[\begin{array}{ccc}
2 & \alpha & \beta \\
2 & -1+\gamma & -1-\gamma \\
2 & -1-\delta & -1+\delta
\end{array}\right]
$$

\subsection{The $Z_{5}$ family}

The general $Z_{5}$ (five-state chiral Potts) model is described by a $5 \times 5$ cyclic matrix. We may consider its 2 -parameter reduction obtained by imposing that the matrix is symmetric. It falls into a family of quasi-integrable models parameterized by a complex number $q$, which (whenever this makes sense) is identifiable with the square root of the number of states [29. The collineation matrix is

$$
C_{Z_{5}}(q)=\left[\begin{array}{ccc}
2 & q^{2}-1 & q^{2}-1 \\
2 & -1+q & -1-q \\
2 & -1-q & -1+q
\end{array}\right]
$$

Its singular set is made of ten points: $P_{1}, P_{2}, P_{3}$ which are the usual singular points of $J, Q_{1}=[1,1,1]$ and $Q_{ \pm}=[-4,1 \pm q, 1 \mp q]$, the singular points of $I$, and $R_{2}=I\left(P_{2}\right), R_{3}=I\left(P_{3}\right), R_{ \pm}=J\left(Q_{ \pm}\right)$. All these points are indetermination points for the invariant

$$
\Delta_{Z_{q}}=\frac{(x-z)(y-z)\left((q-1)\left(x^{2}+y^{2}\right)+2(q+1) x y\right)}{(2+(q-2)(z x+z y)+2 x y)(x-y)^{2}}
$$

Notice that here $S(\Delta)=S(\Gamma)$. The diagram $D_{\Gamma}$ is the following:
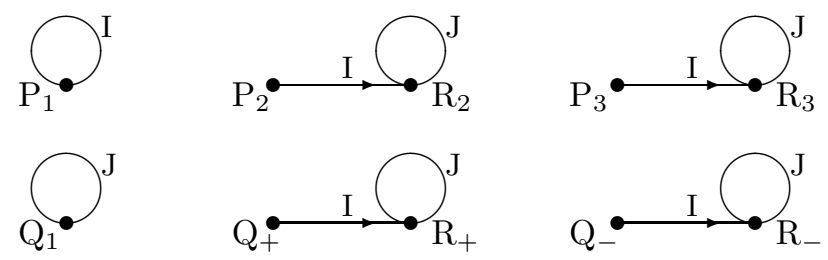


\subsection{The BMV family}

The matrix of (Boltzmann) weights of the BMV model [6] is

$$
\left[\begin{array}{llllll}
x & y & z & y & z & z \\
z & x & y & z & y & z \\
y & z & x & z & z & y \\
y & z & z & x & z & y \\
z & y & z & y & x & z \\
z & z & y & z & y & x
\end{array}\right]
$$

The inversion $I$ is just the matrix inversion. This model pertains to the oneparameter family of quasi-integrable models whose collineation matrix is

$$
C_{B M V}(w)=\left[\begin{array}{ccc}
1 & w-1 & w \\
1 & -1 & 0 \\
1 & 0 & -1
\end{array}\right]
$$

and is reached for the value $w=3$. The singular locus is made out of $P_{1}, P_{2}, P_{3}$ and

$$
Q_{1}=[1,1,1], Q_{2}=[w-1,-(w+1), w-1], Q_{3}=[1,1,-1],
$$

together with an extra point $R=J\left(Q_{2}\right)=\left[w^{2}-1,(w-1)^{2}, w^{2}-1\right]$. The case $w=1$ is singular. The family admits the invariant

$$
\Delta_{B M V}(w)=\frac{P_{w}^{2}(x, y, z) Q_{w}(x, y, z)}{(y+z)^{4}(x-z)^{2}(x-y)}
$$

where

$$
P_{w}(x, y, z)=(1-w)\left(z^{2}-x y\right)+(w-3) z(x-y),
$$

$Q_{w}(x, y, z)=\left(1-w^{2}\right)\left(y^{3}-x z^{2}\right)+\left(w^{2}-4 w-1\right) y^{2}(x-z)+2(w-1)^{2} y z(x-y)$

Here again $S(\Delta)=S(\Gamma)$ and the singular graph is as follows:

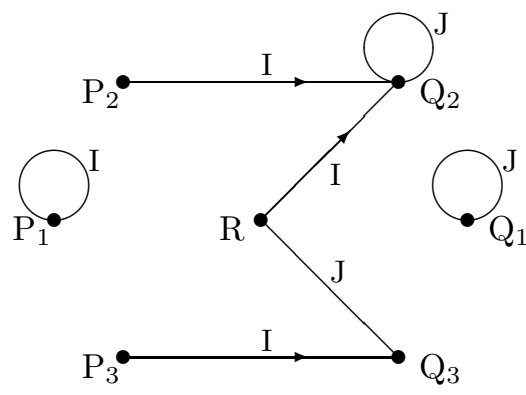




\subsection{The symmetric Ashkin-Teller model}

By symmetric Ashkin-Teller model we understand the 4-state spin model with the cyclic and symmetric matrix of Boltzmann weights

$$
\left[\begin{array}{llll}
x_{0} & x_{1} & x_{2} & x_{1} \\
x_{1} & x_{0} & x_{1} & x_{2} \\
x_{2} & x_{1} & x_{0} & x_{1} \\
x_{1} & x_{2} & x_{1} & x_{0}
\end{array}\right]
$$

for which the matrix inversion is collineated to the Hadamard inversion by means of the matrix

$$
C_{A T}=\left[\begin{array}{ccc}
1 & 2 & 1 \\
1 & 0 & -1 \\
1 & -2 & 1
\end{array}\right]
$$

The singular points of the matrix inversion are $Q_{1}=[1,1,1], Q_{2}=[1,0,-1]$, $Q_{3}=[1,-1,+1]$.

The singular diagram is drawn below
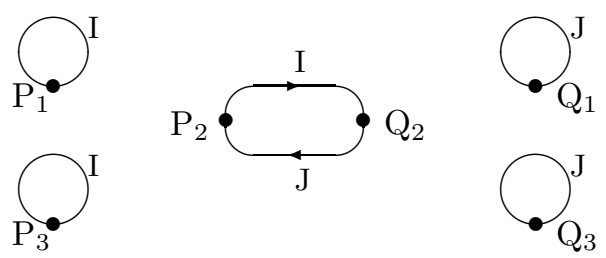

It contains a loop connecting the two points $P_{2}$ and $Q_{2}$. This means that the points $P_{2}=[0,1,0]$ and $Q_{2}=C^{-1}\left(P_{2}\right)$ are not singular for $I J$ and hence it is no surprise that they are non singular for the invariant

$$
\Delta_{A T}=\frac{y^{2}-x z}{y(x-z)} .
$$

There is a strict inclusion of $S(\Delta)$ in $S(\Gamma)$, since the points $P_{2}$ and $Q_{2}$ are not in $S(\Delta)$. The corresponding divisors $\Pi_{P_{2}}=\{$ the line $y=0\}\left(\operatorname{resp} \Pi_{Q_{2}}=\right.$ $\{$ the line $x=z\})$, are indeed curves in the pencil $\Delta=$ const.

\subsection{A finite realization}

It is instructive to consider the group described by the collineation matrix

$$
C_{F}(q)=\left[\begin{array}{ccc}
1 & 0 & 1 \\
1 & q & -(1+q) \\
1 & 0 & -1
\end{array}\right]
$$


which is invertible for $q \neq 0$. It is apparent that $I$ and $J$ share $P_{2}=[0,1,0]$ as a common singular point. Apart from $P_{2}, I$ admits as singular points $Q_{1}=[1,1,1]$ and $Q_{3}=[q,-(2+q),-q]$. The singular graph $D_{\Gamma}$ depicted below contains two more points $R=J\left(Q_{3}\right)$ and $S=I\left(Q_{2}\right)$. This model admits at least two algebraically independent invariants, which can be taken to be

$$
\begin{gathered}
\Delta_{F}^{(1)}=\frac{z^{4}+x^{4}}{z^{2} x^{2}} \\
\Delta_{F}^{(2)}=\frac{q^{2} z x(y-z)^{2}+q\left[\left(z^{2}-x y\right)^{2}-z(z-2 x)\left(x^{2}+y^{2}\right)\right]+2\left(z^{2}-x y\right)^{2}}{(z-y)^{2}\left(z^{2}+x^{2}\right)}
\end{gathered}
$$

This, together with Bezout's theorem tells us that the orbit under $\Gamma$ of any point $p \in \mathbb{P}_{2}$ is finite and of order not greater that 16 , i.e $\Gamma$ is finite. A direct inspection shows that $(I J)^{4}=\mathbf{1}$ and there are additional invariants, of course not algebraically independent from the two previous ones.

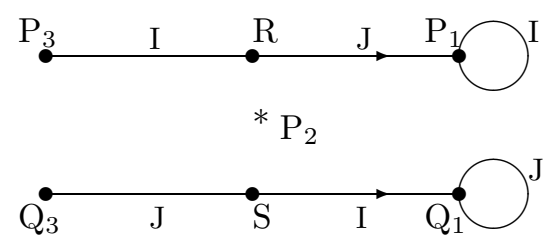

\subsection{The symmetric $Z_{7}$ model}

The symmetric $Z_{7}$ model [6] may be defined by the collineation matrix

$$
C_{Z_{7}}=\left[\begin{array}{ccc}
2 & 6 & 6 \\
2 & -1-i \sqrt{7} & -1+i \sqrt{7} \\
2 & -1+i \sqrt{7} & -1-i \sqrt{7}
\end{array}\right]
$$

From the point of view of dynamical systems the model shows chaotic properties in $\mathbb{P}_{2}$. It has an infinite singular orbit $S(\Gamma)$. According to proposition 5.1 , it does not admit any invariant, as it is confirmed by a direct inspection of the orbits. This model behaves actually like a generic element of the family (9).

\subsection{A finite diagram model (FDM)}

Here $\Gamma$ is generated by the Hadamard inverse $J$ and $I=D^{-1} J D$ with

$$
D=\left[\begin{array}{ccc}
2 & 0 & 2 \\
1 & 1 & -1 \\
-1 & 1 & 1
\end{array}\right]
$$


Notice that $D$ is not of the form (9). The singular diagram is finite but $\Gamma$ does not have any invariant. This provides a remarkable example for what we said in section(5), i.e. that the finiteness of the singular orbit is only a necessary condition for the existence of a non-trivial invariant.

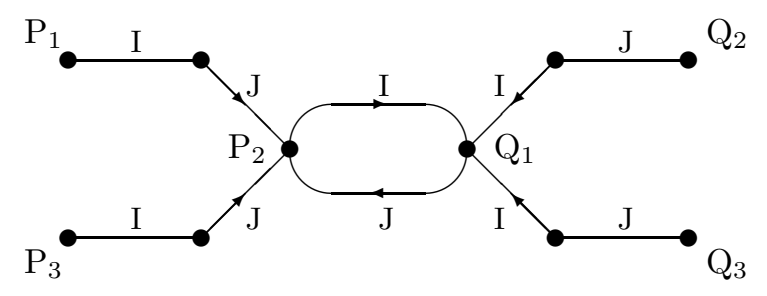

\section{About complexity}

In this section we will discus the notion of complexity of the groups we have been considering.

As we said earlier, we may consider the group $G$ as generated by involutions and relations. If the number $\nu$ of generators is bigger than $2, G$ is "exponentially big" [30]. We will not comment on this here, and limit ourselves to $\nu=2$, as one would concentrate on 1-dimensional subgroups of differentiable groups, and examine the realizations $\Gamma$.

What we want to point out is that there is a very diverse behaviour of these realizations, even in the case $\nu=2$, manifesting itself in the complexity of the representing transformations. The notion of complexity we appeal to is a simple form of the one introduced in [20].

Suppose $F$ is a diffeomorphism of a compact smooth n-manifold $M$. Let $S_{k}$ and $R_{l}$ submanifolds of $M$ of dimension respectively $k$ and $l$. Let $S_{k}^{m}$ be the $\mathrm{m}^{\text {th }}$ iterate of $S_{k}$ by $F$ and $T_{S, R}(m)$ the intersection

$$
T_{S, R}(m)=R_{l} \cap S_{k}^{m} .
$$

Arnol'd [20] defines the complexity $C_{S, R}(m)$ as

$$
C_{S, R}(m)=\sum b_{p}\left(T_{S, R}(m)\right)
$$

the $b_{p}$ 's being the Betti numbers. He also proves that for a sufficiently generic choice of $S_{k}$ and $R_{l}$ the complexity grows at most exponentially with $m$.

The analysis of complexity of plane mappings has already been achieved for the case of polynomial and polynomially invertible transformations [31, 21. We are interested in a wider class of transformations, as the one described in section(5) where $\Gamma$ is generated by two rational transformations. 
Table 1: Behaviour of degrees

\begin{tabular}{|c|c|c|}
\hline Model & Growth & rank of invariants \\
\hline $\mathrm{Z}_{5}$ & $d(k+1)+d(k-1)-2 d(k)$ periodic of period 3 & one \\
BMV & $d(k+1)+d(k-1)-2 d(k)$ bounded & one \\
Finite & $d(k)$ periodic of period 4 & two \\
A-T & $d(k)=4 k$ & one \\
FDM & $d(k)=4^{k}$ (generic) & none \\
$\mathrm{Z}_{7}$ & $d(k+1)-d(k)=f_{2 k}\left(\left\{f_{k}\right\}\right.$ a Fibonacci sequence) & none \\
\hline
\end{tabular}

Although these transformations are not diffeomorphisms there is a natural measure of the complexity by the degree of iterates.

Generically, if the two generating involutions are of degree $u$ and $v$ respectively, the degree of $\varphi=I K$ is $w=u v$, so that $\operatorname{deg} \varphi^{(k)}=w^{k}$. If $\Sigma_{1}$ and $\Sigma_{2}$ are two linear subspaces of $\mathbb{P}_{2}$ then $\varphi^{(k)}\left(\Sigma_{1}\right)$ should be for generic $\Gamma$ a curve of degree $w^{k}$, and the complexity $C_{\Sigma_{1}, \Sigma_{2}}(k)$ would then be exactly $w^{k}$.

However, due to the fact that we are working with projective space, there is a simple mechanism for the lowering of the degree of the iterates $\varphi^{(k)}$, for one has to factorize out common factors from the expressions of the homogeneous coordinates of $\varphi^{(k)}$. This provides a variety of behaviours for the degree $d$ as a function of the order of iteration $k$, lying between exponential growth and periodicity, with the particular case of polynomial (or polynomially bounded) growth.

The outcome of our analysis is that there is a connection between the existence of an invariant and a polynomial (as opposed to exponential) rate of growth, as shows Table 1, inferred from the results of the direct calculation of the first few iterations on the examples of section (6) where the degree of $\varphi$ is 4 .

A more detailed analysis of these properties, together with the study of other properties of realizations of Coxeter groups qua dynamical systems such as finite (periodic) orbits is the matter of further investigations (see [32, 33]).

Acknowledgments We thank M. Talon, O. Babelon, M. Bellon, J-M. Maillard, and G. Rollet for a number of stimulating discussions.

\section{References}

[1] H.S.M. Coxeter and W.O.J. Moser. Generators and relations for discrete groups. Springer Verlag, second edition, (1965).

[2] J.E. Humphreys. Reflection Groups and Coxeter Groups. Cambridge University Press, Cambridge, (1990). 
[3] R.J. Baxter. Exactly solved models in statistical mechanics. London Acad. Press, (1981).

[4] R.J. Baxter, The Inversion Relation Method for Some Two-dimensional Exactly Solved Models in Lattice Statistics. J. Stat. Phys. 28 (1982), pp. $1-41$.

[5] L.D. Faddeev. Integrable models in $1+1$ dimensional quantum field theory. In Les Houches Lectures (1982), Amsterdam, (1984). Elsevier.

[6] M.P. Bellon, J-M. Maillard, and C-M. Viallet, Integrable Coxeter Groups. Physics Letters A 159 (1991), pp. 221-232.

[7] M.P. Bellon, J-M. Maillard, and C-M. Viallet, Higher dimensional mappings. Physics Letters A 159 (1991), pp. 233-244.

[8] M.P. Bellon, J-M. Maillard, and C-M. Viallet, Infinite Discrete Symmetry Group for the Yang-Baxter Equations: Spin models. Physics Letters A 157 (1991), pp. 343-353.

[9] M.P. Bellon, J-M. Maillard, and C-M. Viallet, Infinite Discrete Symmetry Group for the Yang-Baxter Equations: Vertex Models. Phys. Lett. B 260 (1991), pp. 87-100.

[10] M.P. Bellon, J-M. Maillard, and C-M. Viallet, Rational Mappings, Arborescent Iterations, and the Symmetries of Integrability. Physical Review Letters 67 (1991), pp. 1373-1376.

[11] V.F.R Jones. Baxterization. preprint CMA-R23-89, (1989).

[12] H. Poincaré. Les méthodes nouvelles de la mécanique céleste. GauthierVillars, Paris, (1892).

[13] H. Poincaré. Oeuvres. Tomes I-XI. Gauthier-Villars, Paris, (1952).

[14] A.S. Wightman, The mechanics of stochasticity in classical dynamical systems. Perspectives in Statistical Physics (1981), pp. 343-363. Reprinted in "Hamiltonian dynamical systems", R.S. MacKay and J.D. Meiss editors, Adam Hilger (1987).

[15] V.G. Papageorgiou, F.W. Nijhoff, and H.W. Capel, Integrable mappings and nonlinear integrable lattice equations. Phys. Lett. A147 (1990), pp. 106-114.

[16] G.R.W. Quispel, J.A.G. Roberts, and C.J. Thompson, Integrable Mappings and Soliton Equations. Phys. Lett. A 126 (1988), p. 419.

[17] G.R.W. Quispel, J.A.G. Roberts, and C.J. Thompson, Integrable Mappings and Soliton Equations II. Physica D34 (1989), pp. 183-192. 
[18] B. Grammaticos, A. Ramani, and V. Papageorgiou, Do integrable mappings have the Painlevé property? Phys. Rev. Lett. 67 (1991), pp. 1825-1827.

[19] A. Ramani, B. Grammaticos, and J. Hietarinta, Discrete versions of the Painlevé equations. Phys. Rev. Lett. 67 (1991), pp. 1829-1832.

[20] V.I. Arnold, Dynamics of complexity of intersections. Bol. Soc. Bras. Mat. 21 (1990), pp. 1-10.

[21] A.P. Veselov, Growth and Integrability in the Dynamics of Mappings. Comm. Math. Phys. 145 (1992), pp. 181-193.

[22] R.J. Walker. Algebraic Curves. Princeton University Press, Princeton N.J., (1950).

[23] D. Mumford. Algebraic Geometry I: Complex Projective Varieties, volume 221 of Grundlehren der mathematischen Wissenschaften. Springer-Verlag, (1978).

[24] P. Griffiths and J. Harris. Principles of Algebraic Geometry. John Wiley \& Sons, (1978).

[25] I.R. Shafarevich. Basic algebraic geometry. Springer study. Springer, Berlin, (1977). page 216 .

[26] G. Hochschild and J. P. Serre, Cohomology of group extensions. Trans. Am. Math. Soc. 74 (1953), pp. 110-134.

[27] M.P. Bellon, J-M. Maillard, and C-M. Viallet, Quasi integrability of the sixteen-vertex model. Phys. Lett. B 281 (1992), pp. 315-319.

[28] F. Jaeger. Strongly regular graphs and spin models for the Kauffman polynomial. Preprint IMAG, Grenoble, (1991).

[29] M.P. Bellon, J-M. Maillard, G. Rollet, and C-M. Viallet. Deformations of dynamics associated to the chiral Potts model. Preprint PAR-LPTHE 92-17, (1992).

[30] M. Gromov, Groups of polynomial growth and expanding maps. Publ. Math. IHES 53 (1981), pp. 53-73.

[31] S. Friedland and J. Milnor, Dynamical properties of plane polynomial automorphisms. Ergod. Theory Dyn. Systems 9 (1989), pp. 67-99.

[32] M.P. Bellon. Addition on curves and complexity of quasi-integrable rational mappings. in preparation.

[33] S. Boukraa, J-M. Maillard, and G. Rollet. Determinantal identities on integrable mappings. Preprint PAR-LPTHE 92/29, (1992). 